\title{
DIE PFÄNDUNG UND VERWERTUNG DER BUCHAKTIEN IM TÜRKISCHEN ZWANGSVOLLSTRECKUNGSRECHT
}

\author{
Türk İcra-İflas Hukukunda Kaydî Hisselerin Haczi ve Satışı
}

\section{Dr. Gökçen TOPUZ}

I. Einleitung; II. Pfändung der Buchaktien; III. Verwertung der Buchaktien; IV. Fazit

\section{ÖZET}

$\mathrm{Bu}$ çalışmada, menkul kıymetlerin içerdikleri hakların bir senede bağlanmaksızın sadece kaydî olarak ihdas edilmesini ifade eden kaydî hisse, kaydî hissenin haczi ve satışı üzerinde durulacaktır. Kaydî hissede, hissedarlar hissedarlık haklarını ellerinde hisse senedi olmaksızın kaydî olarak bilgisayar ortamında kullanmaktadırlar. Ortada fiziki varlığa sahip her hangi bir senet olmadığı için, kaydî hissenin haczi, fiziki varlığı olan kıymetli evrakın haczinden farklılık arz etmektedir. Çalışmamızda kaydî hissenin İcra ve İflâs Kanunu'nun hangi hükümlerine göre haczedileceği ve paraya çevrilebileceği ele alınacaktır.

Anahtar Kelimeler: Kaydî hisse, hissedar, haciz, Merkezi Kayıt Kuruluşu, paraya çevirme

\footnotetext{
• Ankara Üniversitesi Sosyal Bilimler Enstitüsü Araştırma Görevlisi.
} 


\begin{abstract}
This study focuses on the concept of uncertificated share and its sequestration. Shareholders enjoy their shareholdership rights online, without having any hardcopy documents when uncertificated share is concerned. As there is no hardcopy bill in practice, sequestration of uncertificated shares is different from those of valuable papers which physically exist. This paper analyzes confiscation of uncertificated shares according to Turkish Enforcement and Bankruptcy Act.
\end{abstract}

Keywords: Uncertificated share, shareholder, sequestration, Central Registry Agency (CRA), realization

\title{
I. Einleitung
}

Das Wertrecht bezeichnet nur eine registrierte Verbriefung der im Wertpapier enthaltenen Rechte ohne Anknüpfung an eine Urkunde. Die Aktionäre benutzen beide so genanten Buchaktien, die nicht verbrieft sind und im EDV-System verwahrt werden, ihre daraus entstehenden Rechte buchmäßig im EDV-System ${ }^{1}$. Da kein physisches Wertpapier vorhanden ist, unterscheidet sich die Pfändung die Buchaktien natürlicherweise von der Pfändung die physischen Wertpapiere.

Im türkischen Recht wurde mit dem Art. 10A des Kapitalmarktgesetzes am 15. Dezember 1999 der Bucheffekt angenommen. Der betreffende Artikel lautet mit Gründzügen folgendermaßen:

"Die Instrumente des Kapitalmarktes und die darauf bezogenen Rechte werden nach diesem Artikel durch eine Zentralverwahrungsstelle, welche eine juristische Person des Privatrechts ist, buchmäßig verfolgt. Diese Stelle steht unter die Kontrolle und Aufsicht des Kapitalmarktausschusses. Die Gründungs-, Tätigkeits-, Arbeits- und Aufsichtsgrundlagen der Zentralverwahrungsstelle werden mit einer vom Ministerrat zu verabschiedenden Verwaltungsverordnung bestimmt.

Die Bucheffekten werden von der Zentralverwahrungsstelle im Namen der Emittenten, Makler und Berechtigten buchmäßig eingetragen. Über Bucheffekten werden gemäß dem Art. 7 des Kapitalmarktgesetzes keine Wertpapiere ausgestellt...

Bei der Geltendmachung der Bucheffekten gegenüber den Dritten ist die Ankündigungsdatum an die Zentralverwahrungsstelle maßgeblich ist.

1 Turanboy, Asuman, Varakasız Kıymetli Evrak, Ankara 1998, s.40; Ünal, Oğuz Kürşat, Sermaye Piyasası Hukuku ve Mevzuatı, Ankara 2005, s. 41; Poroy, Reha/ Tekinalp, Ünal, Kıymetli Evrak Hukuku Esasları, İstanbul 2007, s. 42-43; Ülgen, Hüseyin/ Helvac1, Mehmet/ Kendigelen, Abuzer/ Kaya, Aslan, Kiymetli Evrak Hukuku, İstanbul 2007, s. 31; Pulaşlı, Hasan, Kıymetli Evrak Hukuku, Ankara 2007, s. 31-32. 
Bei der im Rahmen der Art. 417 des türkischen Handelsgesetzbuch durch die Mitgliedschaften vorgenommenen Eintragung der Übertragung der Bucheffekte im Aktienbuch ist das von den Emittenten bei der Zentralverwahrungsstelle eingetragene Register ohne Beantragung der Interessenten maßgeblich.

Die Zentralverwahrungsstelle, Emittenten und die Verwahrungsstellen sind wegen eigenen der fehlerhaften Eintragung für die Schäden, die Berechtigten erleiden werden, verschuldensmäßig, verantwortlich...,.

Die Konten, die bei der Zentralverwahrungsstelle (ZWS) bewahrt werden sollen, werden im Namen der Emittenten, Verwahrungsstellen, zuständigen Lagerstellen und Verwahrungsstellen und der vom Kapitalmarktausschuss bestimmten anderen Stellen eröffnet. Die Kontos im Namen der Berechtigten werden nach der Mitteilung deren Personalien an die ZWS eröffnet und mit den Mitgliedern, welche für die Kontogeschäfte zuständig sind, eine Verbindung eingerichtet. Die Geschäfte über die Bucheffekten werden nicht unmittelbar von den Aktionären oder der ZWS, sondern von den Verwahrungsstellen oder Makler vorgenommen. Die Verwahrungsstellen (Makler) üben diese Geschäfte als Vertreter des Berechtigten aus.

\section{Pfändung der Buchaktien}

Die Pfändung von Bucheffekten wird nach den Bestimmungen von Art. 22 der Richtlinie Serie: IV, Nr. $28^{2}$ und Art. 1 der Richtlinie Serie: IV, Nr. $36^{3}$ verwirklicht. Die Regelung in diesen beiden Richtlinien lautet folgendermaßen:

"Die Pfändung der Bucheffekten

Die Pfändung der Bucheffekten wir dadurch vollzogen, dass bei den Kontos betreffenden Anlegern, Teilnehmern, und Mitgliedern ein unter Konto eröffnet wird.

Die Mitglieder sind dazu verpflichtet, vorrangig bei dem Hauptregister bei der ZWS die nötigen Änderungen vorzunehmen, um den zu ihnen gelangten Pfändungsanträgen zu begegnen...,

Angesicht dieser mit beiden Richtlinien vorgenommenen Regelungen wurde die Pfändbarkeit von Bucheffekten akzeptiert. Es ist jedoch nicht klar,

2 Die Richtlinie über die Prozesse und Grundlagen bezüglich der Registrierung der Buchmäßigen Instrumente der Kapitalmarkts, Serie: IV, Nr. 28. In folgenden Teilen dieser Arbeit diese Richtlinie als Richtlinie Serie: IV, Nr. 28.

${ }^{3}$ Die Richtlinie zur Änderung der Richtlinie über die Prozesse und Grundlagen bezüglich der Registrierung der Buchmäßigen Instrumente der Kapitalmarkts, Serie: IV, Nr. 36. In folgenden Teilen dieser Arbeit diese Richtlinie als Richtlinie Serie: IV, Nr. 36. 
nach welchen Bestimmungen die Bucheffekten gepfändet werden. Nach dem letzten Satz des Art. 79 Abs. 2 des türkischen Zwangsvollstreckungs- und Konkursgesetzes (tZKG) "können die im offiziellen Register verzeichneten Sachen von der Zwangsvollstreckungsbehörde durch die Eintragung im Register auch unmittelbar gepfändet werden. , Hier bei ist es wichtig, ob die Hauptregister der ZWS, in dem die Bucheffekten eingetragen sind, im Sinne des Art. 79 tZKG ein offizielles Register ist.

Die ZWS ist nach dem Art. 10/A Abs. 1 des Kapitalmarktgesetzes ein Institut, welches eine juristische Person des Privatrechts ist. Die ZWS wird als eine Aktiengesellschaft gegründet, welche sich im Rahmen des Kapitalmarktgesetzes und der einschlägigen gesetzlichen Bestimmungen betätigt (der Art. 5 Abs. 1 der Verwaltungsverordnung über die GründungsBetätigungs- Arbeits- und Aufsichtsgrundlagen der ZWS).

Die ZWS und ihre Mitglieder sind für die Schäden verantwortlich, die die Berechtigten wegen der fehlerhaften Eintragungen der ZWS und ihrer Mitglieder erleiden (Verwaltungsverordnung Art. 8). Sowohl nach dem Art. 10/A Abs. 1 des Kapitalmarktgesetzes wie auch nach den Art. 5 und 8 der betreffenden Vorschrift ist die ZWS keine juristische Person des öffentlichen Rechts und das Hauptregister bei der ZWS daher kein offizielles Register ${ }^{4}$, weswegen die Buchaktien nach dem Art. 79 Abs. 2 des türkischen Zwangsvollstreckungs- und Konkursgesetzes nicht gepfändet werden dürfen.

Die Buchaktien sind meines Erachtens gemäß dem Art. 89 oder 94 des türkischen Zwangsvollstreckungs- und Konkursgesetzes zu pfänden. Art. 89. lautet: "Wenn Forderungen oder andere Ansprüche, für die nicht eine an den Inhaber lautende oder durch Indossament übertragbare Urkunde besteht, gepfändet werden...,., Die Buchaktien sind im Sinne des Art. 89 "andere Ansprüche“. Zum Begriff “ andere Ansprüche ” im Art. 89 des türkischen Zwangsvollstreckungs- und Konkursgesetzes gehört auch der unverbriefe Aktien des Schuldners an einer Aktiengesellschaft. Aus diesem Grund ist der unverbriefe Aktien auch nach dem Art. 89 pfändbar $^{5}$. Ausgehend von diesem Standpunkt akzeptieren wir, dass auch die Buchaktie zu anderen Ansprüchen zählt. Die Buchaktien werden gemäß Art. 10/A Abs. 2 des Kapitalmarktgesetzes werden nicht beurkundet. Zwar können sie als

\footnotetext{
4 Turanboy, Asuman, 2499 Sayılı Sermaye Piyasası Kanunu'na 4487 Sayılı Kanunla Eklenen 10/A Maddesine Göre Kaydi Değer Düzenlemesi, AÜHFD 1999, C. 48, S. 1-4, s. 46-50; Manavgat, Çağlar, Sermaye Piyasası Kanunu'nun 10/A Maddesi Hükmüne Göre Kaydi Sistemin Esasları, AÜHFD. 2001, C. 50, S. 2, s. 170-173.

${ }^{5}$ Kuru, Baki, İcra ve İflas Hukuku El Kitabı, İstanbul 2004, s. 395; Vgl. Pekcanıtez, Hakan/ Atalay, Oğuz/ Sungurtekin-Özkan, Meral/ Özekes, Muhammet, İcra ve İflâs Hukuku, Ankara 2007, s. 214, dn. 197; Kaçak, Nazif, İcra ve İflâs Kanunu Şerhi, C. 1, Ankara 2006, s. 1010; Uyar, Talih, İcra ve İflâs Kanunu Şerhi, C. 5, İIK 82-97, s. 7853-7854; Süphandağ, Yavuz, İcra ve İflas Hukukunda Uygulamalar, Ankara 2006, s. 264.
} 
Namens- und Inhaberaktien ausgestellt, aber nicht verbrieft werden. Insoweit gibt es bei der Pfändung von Buchaktien kein Problem ${ }^{6}$.

Nach dem Art. 94 des türkischen Zwangsvollstreckungs- und Konkursgesetzes zeigt das Betreibungsamt die Pfändung den beteiligten Dritten an, deren Wohnort bekannt ist, wenn ein Anteil an Gesellschaftsgut oder an einem Gemeinschaftsvermögen gepfändet wird. Da eine Buchaktie einen Anteil an Gesellschaftsgut darstellt ist sie nach dem Art. $94 \mathrm{zu}$ pfänden. Nach der Pfändung der Buchaktien des Schuldners ist den beteiligten Dritten mitzuteilen, dass die Buchaktien des geschuldeten Aktionärs und die aus diesen Buchaktien entstehenden finanziellen Rechte gepfändet wurden. Die Antwort auf die Frage, wer die Dritten sind und an die der im Art. 89 tZKG geregelte Pfändungsbeschluss und die Anzeige nach Art. 94 tZKG zuzustellen sind, ist jedoch unklar.

Meines Erachtens sollte der Dritte, an den der im Art. 89 tZKG geregelte Pfändungsbeschluss und die Anzeige nach dem Art. 94 tZKG sein Makler oder seine Bank sein. Denn die Inhaber der Buchaktien können über die bei der ZWS registrierten Aktien nicht selbst unmittelbar verfügen, sondern sie benutzen ihre Verfügungsbefugnisse durch seinen Makler oder seine Bank. Dies ist nämlich in Art. 6 Abs. 2 und 9 Abs. 2 der Richtlinie Serie IV, Nr. 28 angeführt. Es ist auch möglich, anhand der auf die Pfändung bezogenen Regelungen in den bereits erwähnten Richtlinien zu diesem Schluss zu kommen. Nach Art. 16 der Richtlinie Serie IV, Nr. 36 und einer Änderung des II. Absatzes des Art. 22 der Richtlinie Serie IV, Nr. 28 sind die Mitglieder dazu verpflichtet zur Begegnung von zu ihnen gelangten Pfändungsanträgen vorrangig bei den Eintragung bei der ZWS die notwendigen Änderungen vorzunehmen. Es wir auch geklärt werden, wer der Dritte sein soll an den der Pfändungsbeschluss zuzustellen ist, sofern geklärt wird, wer das Mitglied oder Mitglieder sind, welches/ welche dazu verpflichtet ist/ sind, bei den Eintragung bei der ZWS die notwendigen Änderungen vorzunehmen. Insofern ist es für uns sehr ausschlaggebend, was der Begriff "Mitglied , ausdrückt.

Dieser Begriff bezeichnet nach der Definition im Art. 1 Richtlinie Serie IV, Nr.347, Emittenten, Makler, zuständige Lagerstellen und Verwahrungsstellen sowie von Kapitalmarktausschuss bestimmte andere Institute als Mitglieder. Die Emittenten als Mitglieder der ZWS haben keine Aufgabe, die sie zur Änderung bei dem im ZWS verzeichneten Register übernehmen. Makler und zuständige Lagerstellen und Verwahrungsstellen erfüllen jedoch als Vertreter der Kontoinhaber einige Aufgaben. Nach dem

${ }^{6}$ Dönmez, Murat, Anonim ve Limited Şirketlerde Hisse Haczi ve Paraya Çevrilmesi, İstanbul 2006, s. 60

${ }^{7}$ Die Richtlinie zur Änderung der Richtlinie über die Prozesse und Grundlagen bezüglich der Registrierung der Buchmäßigen Instrumente der Kapitalmarkts, Serie: IV, Nr. 34. In folgenden Teilen dieser Arbeit diese Richtlinie als Richtlinie Serie: IV, Nr. 34. 
Art. 31 Abs. 1 des Kapitalmarktgesetzes dürfen der Verkauf von bei dem Kapitalmarktausschuss zu registrierenden Instrumenten des Kapitalmarkts durch die Emission und das öffentliche Angebot nur von Makler vermittelt und die Kauf- und Verkaufgeschäfte der früher emittierten Instrumenten des Kapitalmarkts als Vermittler wiederum nur von Maklern abgewickelt werden.

Gemäß dieser Regelung darf der Schuldner dessen Buchaktien gepfändet worden sind, dies ausschließlich durch Makler verwirklichen um seine Aktien einem anderen übertragen zu können. Der Pfändungsbeschluss nach der Art. 89 und die Anzeige nach Art. 94 des türkischen Zwangsvollstreckungs- und Konkursgesetzes sind an die Makler zuzustellen, damit diese ihren Zweck erfüllen können. Außerdem kann das Pfändungsbegehren nach Art. 22 Abs. 3 der Richtlinie IV, Nr. 36 unmittelbar an die ZWS mitgeteilt werden.

Durch die an den betreffenden Makler oder ZWS zuzustellende Anzeige muss geteilt werden, dass die Aktien des Schuldners gepfändet worden sind. Die Pfändung in das Register der ZWS umgehend einzutragen ist, weil bei der Geltendmachung der Rechte über die registrierten Instrumenten des Kapitalmarkts gegen die Dritten das Ankündigungsdatum maßgeblich ist, welches von dem betreffenden Mitglied an die ZWS schriftlich oder mit einem von der ZWS bestimmten EDV- Verfahren weitergeleitet werden soll. Die Verfügungsbefugnis des Schuldners über seine gepfändeten Aktien beschränkt ist und daher ohne Zustimmung des Betreibungsamtes und Gläubigers die vorzunehmenden Verfügungen gegen Gläubiger ungültig sind. Der betreffender Makler die Anweisungen des Schuldners über die Nutzung der aus seinen gepfändeten Aktien erfolgenden finanziellen Rechte oder die Übertragung seiner gepfändeten Aktien nicht berücksichtigen bzw. diese Buchaktien lahm legen und blockieren soll. Die aus dem gepfändeten Aktien des Schuldners entstehenden finanziellen Rechte z.B. Gewinne von nun an das Betreibungsamt zu bezahlen sind.

\section{Verwertung der Buchaktien}

Die Verwertung Buchaktien ist nicht im türkischen Zwangsvollstreckungs- und Konkursgesetz geregelt. ZWS hat allerdings mit seinem allgemeinen Brief (Genel Mektup) von 24.11.2005 Nr. 49, die Grundsätze und das Verfahren der Verwertung solche Aktien dargelegt. Danach erfolgt die Verwertung dieser Buchaktien durch die Versteigerung, welche von der Börse (IMKB) veranstaltet wird. Für die Verkaufsvorgänge der Versteigerung wird das Verfahren der Verrechnungsvorgänge entsprechend angewendet. Der Verkauf von Werten, die anhand von der Börse (IMKB) übergegebene Informationen erfolgen, wird für die Abdeckung der Vorgangsschulden des Mitglieds benutzt. Der durch den Mitglied verkaufte Werten erziehen Versteigerungserlös wird in den 
Unterkonto der Pfändung des Mietglieds überwiesen, wodurch diese Unterkonten gestrichen wird. Dieser Versteigerungserlös wird von den Mitglied in den Konto der Betreibungsamt überwiesen. Dieser Konto wird von dem jeweiligen Betreibungsamt bestimmt (ZVS, allgemeine Brief (Genel Mektup) von 24.11.2005 Nr. 49, s. 3).

\section{Fazit}

Die Buchaktien sind gemäß dem Art. 89 oder 94 des türkischen Zwangsvollstreckungs- und Konkursgesetzes zu pfänden. Die Buchaktien sind im Sinne des Art. 89 "andere Ansprüche,.. Die Buchaktie werden gemäß Art. 10/A Abs. 2 des Kapitalmarktgesetzes werden nicht beurkundet. Zwar können sie als Namens- und Inhaberaktien ausgestellt, aber nicht verbrieft werden. Insoweit gibt es bei der Pfändung von Buchaktien kein Problem. Nach dem Art. 94 des türkischen Zwangsvollstreckungs- und Konkursgesetzes zeigt das Betreibungsamt die Pfändung den beteiligten Dritten an, deren Wohnort bekannt ist, wenn ein Anteil an Gesellschaftsgut oder an einem Gemeinschaftsvermögen gepfändet wird. Da eine Buchaktie einen Anteil an Gesellschaftsgut darstellt ist sie nach dem Art. 94 zu pfänden. Die Verwertung solcher Aktien ist nicht im türkischen Zwangsvollstreckungs- und Konkursgesetz geregelt. ZWS hat allerdings mit seinem allgemeinen Brief von 24.11.2005 Nr. 49, die Grundsätze und das Verfahren der Verwertung solche Aktien dargelegt. Danach erfolgt die Verwertung dieser Buchaktien durch die Versteigerung, welche von der Börse (IMKB) veranstaltet wird. 


\section{Abkürzungsverzeichnis}

EDV : Elektronische Datenverarbeitung

IMKB : İstanbul Menkul Kıymetler Borsası

tZKG : türkischen Zwangsvollstreckungs- und Konkursgesetzes

ZVS: Zentralverwahrungsstelle

\section{Literaturverzeichnis}

Dönmez, Murat:Anonim ve Limited Şirketlerde Hisse Haczi ve Paraya Çevrilmesi, İstanbul 2006.

Kuru, Baki : İcra ve İflas Hukuku El Kitabı, İstanbul 2004.

Kaçak, Nazif : İcra ve İflâs Kanunu Şerhi, C. 1, Ankara 2006.

Manavgat, Çağlar : Sermaye Piyasası Kanunu'nun 10/A Maddesi Hükmüne Göre Kaydi Sistemin Esasları, AÜHFD. 2001, C. 50, S. 2.

Pekcanıtez, Hakan/Atalay, Oğuz/Sungurtekin-Özkan, Meral/Özekes, Muhammet : İcra ve İflâs Hukuku, Ankara 2007.

Poroy, Reha/Tekinalp, Ünal : Kıymetli Evrak Hukuku Esasları, İstanbul 2007.

Pulaşl1, Hasan : Kıymetli Evrak Hukuku, Ankara 2007.

Süphandağ, Yavuz : İcra ve İflas Hukukunda Uygulamalar, Ankara 2006.

Turanboy, Asuman : Varakasız Kıymetli Evrak, Ankara 1998.

Turanboy, Asuman : 2499 Sayılı Sermaye Piyasası Kanunu'na 4487 Sayılı Kanunla Eklenen 10/A Maddesine Göre Kaydi Değer Düzenlemesi, AÜHFD 1999, C. 48, S. 1-4.

Uyar, Talih : İcra ve İflâs Kanunu Şerhi, C. 5, İIK 82-97, Ankara 2006.

Ülgen, Hüseyin/Helvac1, Mehmet/Kendigelen, Abuzer/Kaya, Aslan : Kiymetli Evrak Hukuku, İstanbul 2007.

Ünal, Oğuz Kürşat : Sermaye Piyasası Hukuku ve Mevzuatı, Ankara 2005. 